\title{
Neural networks on chemically patterned 'cultured probe' electrode arrays: network growth and activity patterns
}

\author{
W.L.C. Rutten, T.G. Ruardij, B.R.M. van Buul and B.H. Roelofsen
}

Faculty of Electrical Engineering/Institute for Biomedical Technology, University of Twente, Enschede, The Netherlands

\begin{abstract}
A 'cultured probe' is a hybrid type of neural information transducer or prosthesis, for stimulation and/or recording of neural activity in the brain or the spinal cord (ventral motor region or dorsal sensory region). It consists of a micro electrode array (MEA) on a planar substrate, each electrode being covered and surrounded by a locally confined network of cultured neurons, obtained by chemical patterning of the substrate. The purpose of the cultured cells is that they act as intermediates for collateral sprouts from the in vivo system, thus allowing for an effective and selective neuron electrode interface. As the local neural network will become spontaneously active and has the capability of information processing, one may envisage future applications of these intermediary networks as 'front-end' signal processors.

Two aspects of the development of this kind of cultured probe device are described. First, it is shown how substrates can be chemically modified to confine developing networks, cultured from dissociated rat cortex cells, to the surrounding of an electrode site. Secondly, the paper presents results on neuronal activity in such confined, circular networks and synchronized activity between two such interconnected networks.
\end{abstract}

Keywords - Neural networks, network patterning, network activity

\section{INTRODUCTION}

As there are no 'blueprints' for the exact positions of fibres in a peripheral nerve, or motor neurons in a ventral root region, an insertable multielectrode has to be designed in a redundant way. Even then, the efficiency of a multielectrode will be less than $100 \%$, as not every electrode will contact one neural axon or soma.

Therefore, 'cultured probe' devices are being developed, based on cell-cultured planar MEA's (Multi Electrode Arrays). They may enhance efficiency and selectivity because neural cells have been grown over and around each electrode site as electrode-specific local networks. If, after implantation, collateral sprouts branch from a motor fibre (ventral horn area) and if they can be guided and contacted to each 'host' network, a very selective and efficient stimulatory interface will result (Fig. 1).

Essential properties of the probe are that cells must adhere to the surface of the substrate and form networks of somata and outgrowing neurites/axonal fascicles, within a predefined area around the electrodes. For that, chemical modification of the substrate and electrode areas with various coatings, such as polyethyleneimine (PEI) and fluorocarbon (FC) monolayers promotes or inhibits adhesion of cells

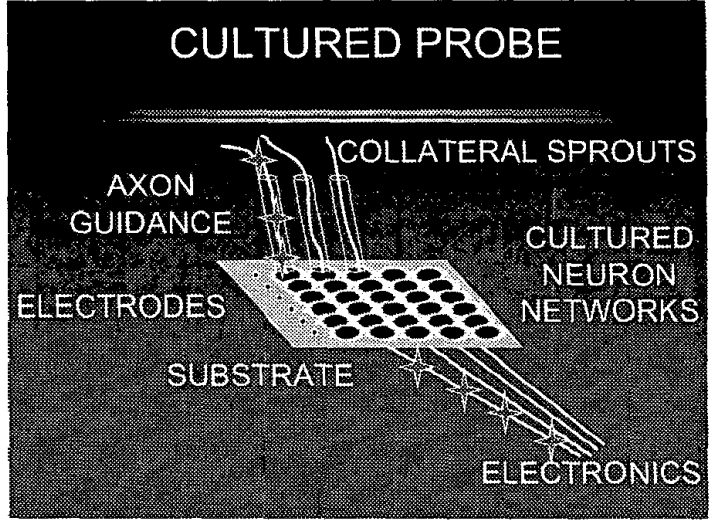

Fig. 1. Schematic impression of a 'cultured probe'-type of neural information transducer/prosthetic device. The black circular 'stand-aloneislands' are cultured neuronal networks at and around each electrode.

One of the key problems in long-term neuronal patterning studies on MEAs is the biofouling of the background material with cell-adhesive proteins, which on turn promote the random overgrowth with neuronal tissue [1]. Polyethylenoxide (PEO)-coated surfaces are known for their ability to inhibit the adsorption of proteins and are promising alternatives as neurophobic background surfaces. The methods to fabricate PEO-coated surfaces can be divided in two different subgroups e.g. covalent bonding of relatively short polyethylenoxide chains (PEO also termed polyethylenglycol PEG) and adsorption of polyethylenoxidepolypropylenoxide (PEO-PPO) block-copolymers onto hydrophobic materials [2] The advantage of covalent coupling of PEO chains to surfaces is the initial stability of the layer and the prohibited displacement of PEO by proteins in solution. However, the chemical bonds between the PEO chains and the underlying substratum could be dissociated by the physiological surrounding in time. Another important point is the fact that the chemistry involved should be transferable onto multi-electrode arrays, which are usually vulnerable to more laborious chemistry. For instance, cleaning of MEAs with aggressive acids before chemical modification is prohibited because the conductive metal leads would be dissolved. Therefore, it is relevant to test more simple modification routes as potential methods to be used on MEAs. Thus, adsorbed layers of PEO-PPO blockcopolymers on hydrophobic surfaces were investigated as potential neurophobic background surfaces in patterning studies, over a time period of 30 days (commercially 


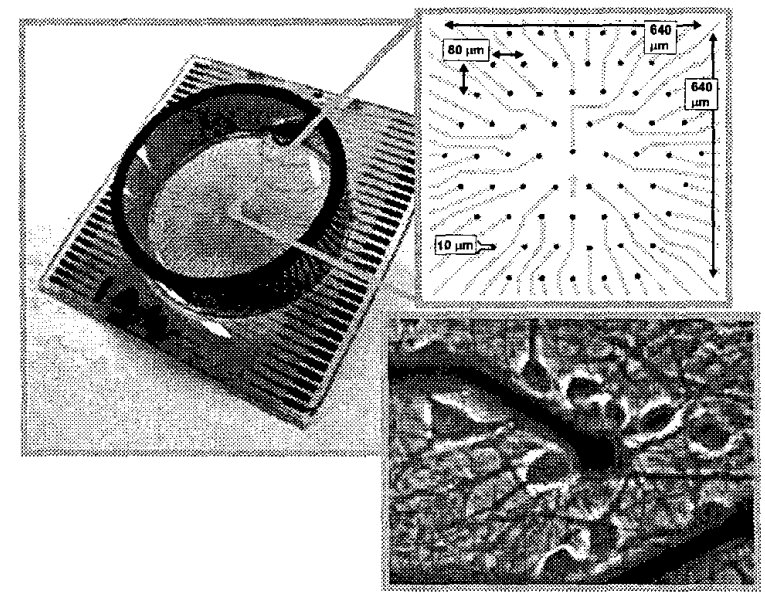

Fig. 2. The culture dish (diameter $30 \mathrm{~mm}$ ) is shown on the left. It is based on a glass substrate $(5 \times 5 \mathrm{~cm})$ with 61 embedded electrodes. The 61 tips (tip diameter $10 \mu \mathrm{m}$ ) lie in the center of the chamber (magnified in right-top figure). The figure right below shows a detail of one tip, surrounded by a 4 DIV old developing cortical cell culture.

available PEO-PPO-PEO triblockcopolymers, called Synperonics F108 and F127 were tried).

Neuronal cultures from rat dissociated cortical cells can be cultured on Micro Electrode Arrays (MEA's, with 61 electrode sites, $12 \mu \mathrm{m}$ diameter) on the bottom of a culture dish (figure 2). Developing neural networks will start to exhibit spontaneous activity after about one week in vitro. To study this we have developed quantitative techniques for long-term, longitudinal recording of activity levels in individual neurons. It has been shown that the patterns resemble those seen in vivo [3]. Recording of the firing activity of individual neurons, has become established, since the pioneering work of Gross and Pine [4], [5] as a useful technique, as is also shown by Jimbo et al. [6] in a study of activity-dependent plasticity at the synaptic level. It can be expected that waveform depends on the position of a soma or axon with respect to the electrode site. Complete covering of a site by a soma is, electrically, quite different from partial coverage, or non-coverage. Elsewhere, the classification of recorded waveforms into a spike waveform database was reported [7].

In the present paper, bakground adhesion results are shown over a period of 30 days for neurophobic, Polyimid and Fluorocarbon coated surfaces, each supplemented by F108 or F127 Synperonics.

Then, we present the occurrence and development of activity in patterned circular island-networks on a cultured probe MEA design (see Figs. 1 and 2).
It is of great interest to answer such questions as: do islands also become spontaneously active after a week ? with the same characteristics as unpatterned (random) networks ? is there a minimum diameter, above which networks become spontaneously active? if stand-alone networks become interconnected, do firing patterns synchronize?

\section{METHODOLOGY}

\section{A. Cortical neuron isolatio/culturing and MEA fabrication}

Dissociated (Trypsin/EDTA) cortical neurons (neonatal rat cerebellum) were seeded onto patterned structures with a plating density of 5000 living cells $/ \mathrm{mm}^{2}$. Cells were allowed to adhere onto the surfaces during a time period of 4 hours. Samples were rinsed with $\mathrm{NaCl}(0.9 \%)$ solution to remove non-adherent cells. Neurons were cultured in R12 medium (DMEM/HAM's F12, Gibco) without serum. The cultures were stored in a $\mathrm{CO}_{2}$ incubator with a constant temperature of $37^{\circ} \mathrm{C}$, and a constant $\mathrm{CO}_{2}$ level of $5 \%$. The culture medium was refreshed half, 3 times a week.

In short, multi-electrode areas (MEAs) were fabricated from $5 \times 5 \mathrm{~cm}$ glass plates with gold deposited wires leading to 61 hexagonal ordered electrodes. MEAs were isolated with a sandwich layer of $\mathrm{SiO}_{2}-\mathrm{Si}_{3} \mathrm{~N}_{4}-\mathrm{SiO}_{2}(\mathrm{ONO})$ using a PECVD process. Electrode tips were deinsulated with a $\mathrm{SF}_{6}$ Reactive Ion Etching (RIE) technique and platinised to reduce the electrode impedance down to $200 \mathrm{k} \Omega$ at $1 \mathrm{kHz}$.

Neurophilic isalnds wre created by PEI microstamping of circular patterns.

\section{B. Quantification and classification of spike waveforms}

Electrode signals were amplified, filtered between $0.3 \mathrm{kHz}$ and $6 \mathrm{kHz}$ (first order) and captured by a 12 bit National Instruments PCI-6023E Data Acquisition PC-card. The input range as well as the sampling frequency was software controlled by a Labview program. The real time data processing software reduced the data stream by rejection of data which does not contain bioelectrical activity. Artefact rejection was severe: if activity is measured at the same time in different channels, the waveforms are rejected. In each channel, the rms noise level was constantly monitored and determines the setting of a level detector to detect spike activity. The threshold was set at 6 times noise level (typically $7 \mu \mathrm{V} \mathrm{rms}$ ). Each time bin of $10 \mathrm{~ms}$ with recorded activity was stored and analysed with Matlab computer software.

Three spike features are the peak-to-peak amplitude Vpp, the width of the peak and the area of the peak. A fourth feature, called peak balance, distinguishes different shapes. It calculates the difference between the waveform area before and after the peak value. Classification procedures have been described elsewhere [7]. 


\section{Fabrication of neurophobic background materials}

1) Polyimide: Polyimide (PI, Probimide $7510^{B}$, Arch Chemicals N.V., Zwijndrecht, Belgium) was spin-coated (4000 rpm, 30 seconds) onto $25 \mathrm{~cm}^{2}$ glass plates (Glaverbel, Belgium). PI was diluted in n-methyl pyrolidon $(1: 1 \mathrm{v} / \mathrm{v})$, dried on a hot plate $\left(120^{\circ} \mathrm{C}, 5\right.$ minutes), exposed to UV-light, and baked $\left(300^{\circ} \mathrm{C}, 90\right.$ minutes). Then plates were cut into square pieces of approximately $2.6 \mathrm{~cm}^{2}$.

2) Plasma-coated Fluorocarbon ( $F C$ ): In a reactive ion etching (RIE) system, spin-coated polyimide samples were treated with an etching $\mathrm{CHF}_{3} / \mathrm{O}_{2}$ plasma $\left(25 \mathrm{sccm} \mathrm{CHF}_{3}, 5\right.$ sccm $\mathrm{O}_{2}, 150 \mathrm{mT}$ orr and $2.1^{*} 10^{-1} \mathrm{~W} / \mathrm{cm}^{2}$ ) for 20 seconds, a depositing $\mathrm{CHF}_{3}$ plasma (25 sccm $\mathrm{CHF}_{3}, 150 \mathrm{mT}$ Torr, and $2.1^{*} 10^{-1} \mathrm{~W} / \mathrm{cm}^{2}$ ) for 40 seconds, and a final depositing $\mathrm{CHF}_{3}$ plasma treatment at $1.2 * 10^{-1} \mathrm{~W} / \mathrm{cm}^{2}$ for 8 minutes.

3) Synperonics F108 and F127: The triblockcopolymers Synperonics F108 $\left(\mathrm{EO}_{127}-\mathrm{PO}_{48}-\mathrm{EO}_{127}\right.$; ICI, Holland BV, Rozenburg) and F127 (EO ${ }_{95}-\mathrm{PO}_{62}-\mathrm{EO}_{95}$; ICI, Holland BV, Rozenburg) were dissolved in $0.1 \mathrm{M}$ phosphate buffered saline $(1 \% \mathrm{w} / \mathrm{w})$ and adsorbed onto Polyimide- and Plasmacoated FC samples over a time period of 24 hours. Subsequently, samples were rinsed twice with sterile water (Aqua Purificata, Bufa BV, Uitgeest, The Netherlands). Surfaces were dried by aspiration of residual water with a glass pipette connected to a vacuum pump.

\section{Quantification of background adhesion}

Microphotographs were taken on 2 separate subsections of each pattern after $1,4,8,15$ and 30 days with a digital photo camera (AxioCam HR, Carl Zeiss, Germany) attached to an inverted phase contrast microscope (Nikon Diaphot-TMD, Tokyo, Japan). The images were first corrected for nonuniform illumination. Secondly, they were converted into black-and-white images by a threshold operation: white pixels for the cell areas, black for the background material. The calculated fraction $F$ of white pixels in the image now represented a quantitative measure for the adhesion on the background material only

$$
\mathrm{F}=\frac{\text { Number of white pixels }}{\text { Number of white pixels }+ \text { black pixels }}
$$

\section{RESULTS}

Figure 4 shows the results of cell adhesion in the neurophobic (called 'background') areas.

The conclusion from figure 4 is that FC coating is better than PI coating, also after 30 DIV. Additional coating with triblock-copolymers, synperonics F108 and F127, repels cells even better, for both PI and FC, but not after 30 days in the F108-FC case. Best results are obtained for the PI-basic layer, with finally only a few \% of neurophobic layer area taken by cells (see [8] for full report)

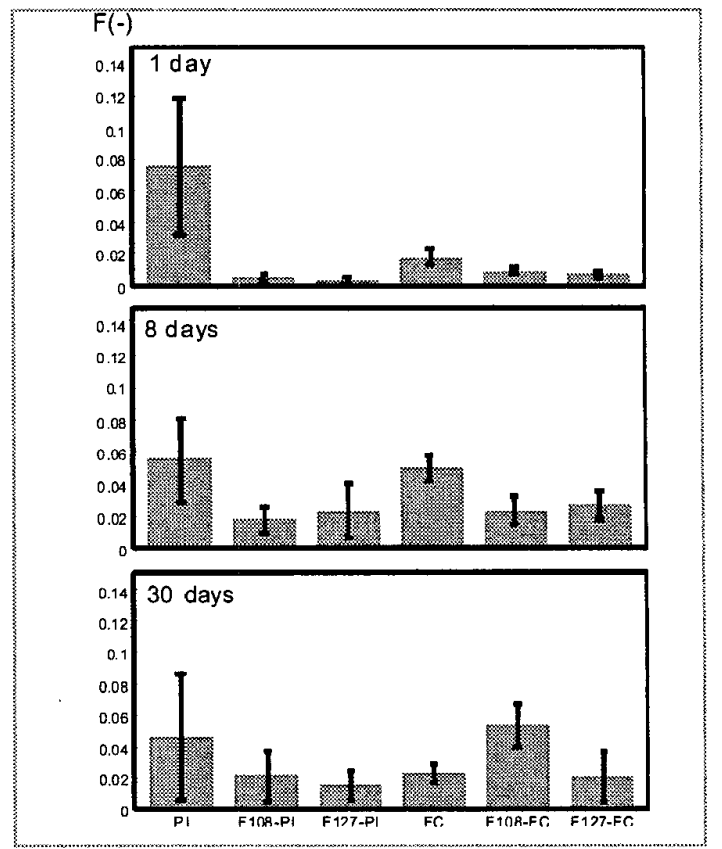

Fig. 4 Quantified background adhesion fraction F (see section II D) of neuronal tissue on different background surfaces (neurophobic part) after 1 , 8 and 30 days. Mean \pm S.D $(n=6)$.

On 1 MEA, with unconnected islands, spike rates in a particular island were observed after 22 DIV. Neurons fired at a rate of 8 spikes / sec at $22 \mathrm{DIV}$, and $4-5$ spikes/s at 28 DIV.

A second, more systematic, search for spontaneous activity in patterned, but connected, islands was done on 2 MEA's,

40

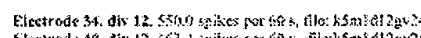

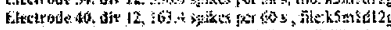
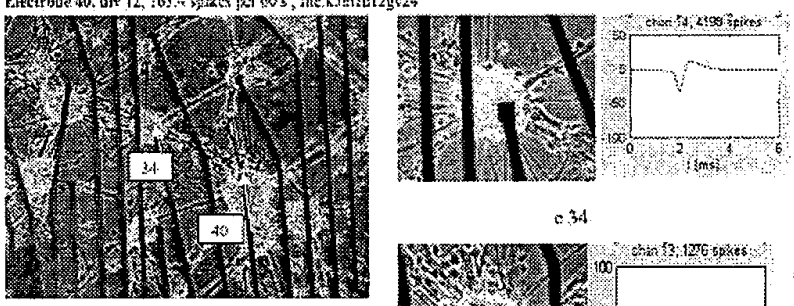

0.4

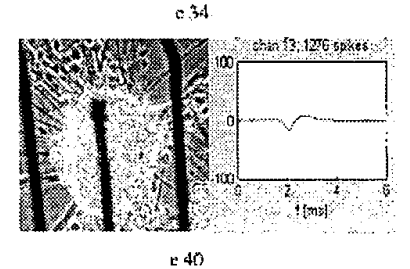

Fig.5. Example of spontaneous bioelectrical activity from cortical neurons seeded onto a PEI-microprinted multi-electrode array (12 DIV). Two islands at electrodes 34 and 40 were spontaneously active after a week Electrode separation is $190 \mu \mathrm{m}$. Islands became interconnected later and showed synchronized behaviour (fig. 6). Details on the right show the two clusters and the derived averaged spike waveform (top: electrode 40,4198 spikes. bottom: el. 34,1276 spikes). 
each with 60 circles of PEI, diameters 50, 100 ad $150 \mu \mathrm{m}$ (20 each). Activity started at $8 \mathrm{DIV}$ and could last until the last observation day, $35 \mathrm{DIV}$. No activity was seen for the $50 \mu \mathrm{m}$ diameter islands. Five $\%$ of the $100 \mu \mathrm{m}$ diameter islands showed activity and $8.5 \%$ of the $150 \mu \mathrm{m}$ diameter islands. Spike rates were much higher than in the unconnected island, ranging from averages (+/ $1 \mathrm{SD})$ of 17 (12), 302 (199), 229 (192), $95(-)$ and $248(-)$, at $8,12,14,19$ and $35 \mathrm{DIV}$, respectively (number of active electrodes/islands was $5,3,4$, 1 , and 1 , respectively).

Figure 5 gives a typical example of two connected islands at 12 DIV. Figure 6 presents the spike rates of these islands, first as a spike rate plot over a time interval of 120 seconds then as a inter electrode interval histogram. It is clearly observed that the two clusters fire in a highly synchronized way, with periodic bursting. In the peaks, spike rates go up to $30 \mathrm{spikes} / \mathrm{sec}$ in one channel, and about $20 \mathrm{spikes} / \mathrm{sec}$ in the other.

Overall, the spike rates are much higher in the connected islands than in the unconnected ones (with the same diameter).
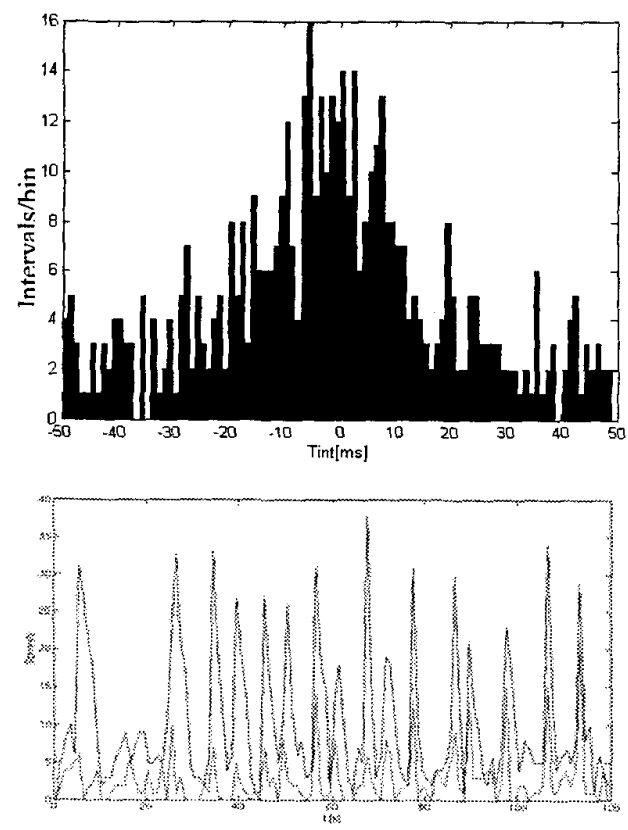

Fig. 6. Top: Spikes/second during 120 seconds of the two interconnected islands of fig. 5. Vertical scale: spikes/second. The two curves are for electrode 34 and 40 (see fig. 5) Spearman correlation coefficient $\rho=0.61$. Below Inter electrode interval histogram, bin-width is $1 \mathrm{~ms}$. Both figures illustrate clearly the periodic bursting of activity, as well as strong synchronization between the island-networks.

\section{COMPARISON TO UNPATTERNED NETWORK}

Activity in patterned MEA's will be briefly compared to unpatterned (random) network activity, as reported in [9].In the latter, activity starts around one week after seeding, i.e. 7
DIV. Spike rate, summed over all 61 electrodes, develops gradually from zero to 32 spikes/sec between 9 and 42 DIV. This implies a maximum of about 0.5 spikes $/ \mathrm{sec}$ per electrode, at 42 DIV.

However, in time, and per electrode, spike rate may differ considerably, for example 22 spikes/s was observed maximally, in one electrode (lasting a few days).

Comparing unpatterned networks with patterned islands, activity seems to start at the same age, 1 week DIV. The variability in the random networks, and absence of statisctics sofar in the unconnected island case, makes it hard to draw a comparison. A cautious observation may be that the spike rate in unconnected islands is in the same range as in the random network, i.e. $4-8$ spikes/s at 22-28 DIV.

It is however clear that the connected clusters fire at a very high spike rate, much higher than observed in the unpatterned network [9].

\section{CONCLUSIONS}

- triblock copolymer coating, on top of basic neurophobic PI or FC coating, enhances neural repulsion, and is still still present at 30 'DIV

- spontaneous activity in cultured neural networks is absent in circular $50 \mu \mathrm{m}$ diameter islands, but present in $100 \mu \mathrm{m}$ (or higher) diameter islands. It starts at normal age, after a week in culture.

- connected islands may show highly synchronized activity, with high spike rates

\section{REFERENCES}

[1] J.M. Corey, B.C. Wheeler and G.J. Brewer, "Micrometer resolution silane-based patterning of hippocampal neurons: critical variables in photoresist and laser ablation processes for substrate fabrication," IEEE Trans. Biomed. Eng, vol. 43, pp. 944-955, 1996.

[2] J.H. Lee, Y.M. Ju, and D.M. Kim, "Platelet adhesion onto segmented polyurethane film surfaces modified by addition and crosslinking of PEOcontaining block copolymers," Biomaterials, vol. 21, pp. 683-691, 2000.

[3] M.A. Corner and G.J.A. Ramakers, "Spontaneous firing as an epigenetic factor in brain development - physiological consequences of chronic tetrodotoxin and picrotoxin exposure in cultured rat neocortex neurons". Dev Brain Res, 65:57-64, 1992.

[4] G. Gross, "Simultaneous single unit recording in vitro with a photoetched laser deinsulated gold multielectrode surface", IEEE TransBiomed Eng, 26:273-278, 1979.

[5] J. Pine "Recording action potentials from cultured neurons with extracellular microcircuit electrodes" J Neurosci Meth , 2:1931,1980

[6] Y. Jimbo, T. Tateno, and H.P.C. Robinson, "Simultaneous induction of pathway-specific potentiation and depression in networks of cortical neurons", Biophys $J, 76: 670-678,1999$.

[7] G.W. van Staveren, J.R. Buitenweg, T. Heida, and W.L.C. Rutten, "Wave shape classification of spontaneous neuronal activity in cortical cultures on micro-electrode arrays", Proceedings second joint IEEEEMBS/BMES conference, Houston USA, ISBN: 0-7803-7613-7 CD-ROM. 2002.

[8] T.G. Ruardij, M.A.F. van den Boogaart and W.L.C. Rutten, "Adhesion and growth of electrically-active cortical neurons on polyethyleneimine patterns microprinted on PEO-PPO-PEO triblockcopolymer-coated hydrophobic surfaces", IEEE Transactions on Nanobioscience, vol 1(1), 1$8,2002$.

[9] W.L.C.Rutten, and J. van Pelt, "Activity patterns of cultured neural networks on micro electrode arrays". Proceedings $23 \mathrm{rd}$ International Conference of the IEEE Engineering in Medicine and Biology Society, Istanbul, Turkey. 4 pp. ISBN 0-7803-7213-1 CDROM, 2001. 